\title{
$B A G E$ Hypomethylation Is an Early Event in Colon Transformation and Is Frequent in Histologically Advanced Adenomas
}

\author{
Erica Lana ${ }^{1}$, Marie-Elisabeth Brun ${ }^{2}$, Isabelle Rivals ${ }^{3}$, Janick Selves ${ }^{4}$, Sylvain Kirzin ${ }^{4}$, Andriy \\ P. Lutsyk ${ }^{5}$, Vasily V. Gordiyuk ${ }^{6}$, Frédéric Bibeau ${ }^{7}$, Alla Rynditch ${ }^{6}$ and Albertina De Sario ${ }^{1, *}$ \\ 1 INSERM U827, Montpellier, France; E-Mail: erica.lana@inserm.fr (E.L.) \\ 2 Institut de Génétique Humaine, CNRS UPR 1142, Montpellier, France; \\ E-Mail: elisabeth.brun@igh.cnrs.fr \\ 3 Equipe de Statistique Appliquée, ESPCI ParisTech, Paris, France; E-Mail: isabelle.rivals@espci.fr \\ 4 CHU Purpan and INSERM U563, Toulouse, France; E-Mail: selves.j@ chu-toulouse.fr (J.S.), \\ kirzin.s@chu-toulouse.fr (S.K.) \\ 5 O.O. Bogomoletz National Medical University, Kyiv, Ukraine; E-Mails: alutsyk@gmail.com \\ 6 Department of Functional Genomics, Institute of Molecular Biology and Genetics NASU, Kyiv, \\ Ukraine; E-Mail: vasilij_gordiyuk@yahoo.com (V.V.G.), rynditch@imbg.org.ua (A.R.) \\ 7 CRLC, Montpellier, France; E-Mail: frederic.bibeau@valdorel.fnclcc.fr \\ * Author to whom correspondence should be addressed; E-Mail: albertina.de-sario@inserm.fr; \\ Tel.: +33-467-415-360; Fax: +33-467-415-365.
}

Received: 27 September 2009; in revised form: 28 October 2009 / Accepted: 13 November 2009 / Published: 18 November 2009

\begin{abstract}
We showed earlier that BAGE (B melanoma antigen) loci are hypermethylated in normal tissues and hypomethylated in $98 \%$ of human cancers. More recently, we provided evidence that hypomethylation of $B A G E$ loci represents an informative marker for colon cancer detection. In this study, we show that hypomethylation of BAGE loci was an early event that occurred in $43 \%$ of colorectal adenomas. Interestingly, hypomethylation of $B A G E$ loci was frequent $(50 \%)$ in tubulo-villous and villous adenomas, these adenomas having a high probability of being transformed into colorectal cancers.
\end{abstract}

Keywords: $B A G E$; DNA hypomethylation; adenomas; colorectal cancer; biomarker; COBRA 


\section{Introduction}

Colorectal cancer is the third most common cancer worldwide and is the third leading cause of cancer-related deaths. Currently detection of precancerous lesions and cancers at an early stage seems to be the most effective means of reducing mortality. Several colorectal screening tests are available. Fecal occult blood testing, a noninvasive test used to screen asymptomatic subjects that are 50 years or older, has a limited sensitivity [1]. Colonoscopy and sigmoidoscopy are more sensitive, but cannot be used routinely because of high costs and discomfort for the patient. Therefore, optimized screening methods should be established to monitor the general population.

Colorectal cancer results from a multistep process comprising several histological changes, the so called adenoma-carcinoma progression sequence of events [2]. Both genetic and epigenetic mutations associated with these histological changes have been described. Some of these mutations clearly contribute to the transformation process; for others, a pathogenic role is not yet proven. Genetic and epigenetic mutations that are frequently associated with cancer cells can be used as biological markers for cancer diagnosis. Mutations that are already detected in precancerous lesions are the most valuable tumor markers because they allow patients to be treated before they develop metastases.

$B A G E$ loci are a family of full-length and truncated genes located in the juxtacentromeric heterochromatic regions of several human chromosomes [3,4]. BAGE genes are silent in normal tissues and expressed in some tumors and in male germ cells, whereas truncated genes are nonfunctional. We showed earlier that $B A G E$ loci (i.e. genes and truncated genes) are hypermethylated in normal tissues and hypomethylated in $98 \%$ of human cancers [5]. More recently, we have shown that $B A G E$ loci are hypomethylated in $83 \%$ of colon cancers. Using the methylation of $B A G E$ loci as tumor marker, colon cancer could be diagnosed with $94 \%$ specificity, $83 \%$ sensitivity, and $89 \%$ accuracy [6]. In the present study, we asked whether $B A G E$ hypomethylation can be used to detect colorectal precancerous lesions.

\section{Results and Discussion}

\subsection{BAGE loci Are Hypomethylated in Colorectal Adenomas}

We showed earlier that $B A G E$ loci are hypermethylated in normal tissues and hypomethylated in $83 \%$ of colon cancers [6]. To determine whether loss of methylation of BAGE loci is an early event in colon transformation, we have analyzed 44 colorectal adenomas (Table 1 and Figure 1) using the COBRA assay that we described earlier [5]. We showed previously that a DNA methylation threshold of $42 \%$ made it possible to distinguish colon cancer from normal mucosa with $94 \%$ specificity and $83 \%$ sensitivity [6]. In the present study, using the same threshold, we found that BAGE loci are hypomethylated in 19 out of $44(43 \%)$ adenomas (Table 1 and Figure 1). The percentage of precancerous cells in our samples ranged from $60 \%$ to $100 \%$ (Table1). DNA hypomethylation did not correlate with the percentage of precancereous cells observed in adenomas (Spearman test $r=-0.01$, $\mathrm{p}=0.97$ ). Our samples were collected in two different geographical areas: France and Ukraine. In spite of possible ethnic differences in the two patient populations, the prevalence of $B A G E$ hypomethylation in colon adenomas was similar: 42\% $(\mathrm{n}=24)$ in France and $45 \%(\mathrm{n}=20)$ in Ukraine (Fisher's exact test, two-sided, $\mathrm{p}=1$ ). To conclude, our results show that loss of methylation in BAGE loci is an early event in colon transformation. 
No correlation was found between DNA methylation and age (Spearman's $r=-0.04, p=0.82$ ) and sex (Wilcoxon test, $\mathrm{p}=0.63$ ) of patients, nor with the location of the adenomas (Kruskal-Wallis test, $\mathrm{p}=0.76)$.

Figure 1. DNA methylation percentages in five tissues. Data on paired colon cancer and healthy mucosas are from our previous study [6]; whereas data on adenomas are from this study (a different cohort of patients). T are tubulous, TV are tubulo- villous and $\mathrm{V}$ are villous adenomas.

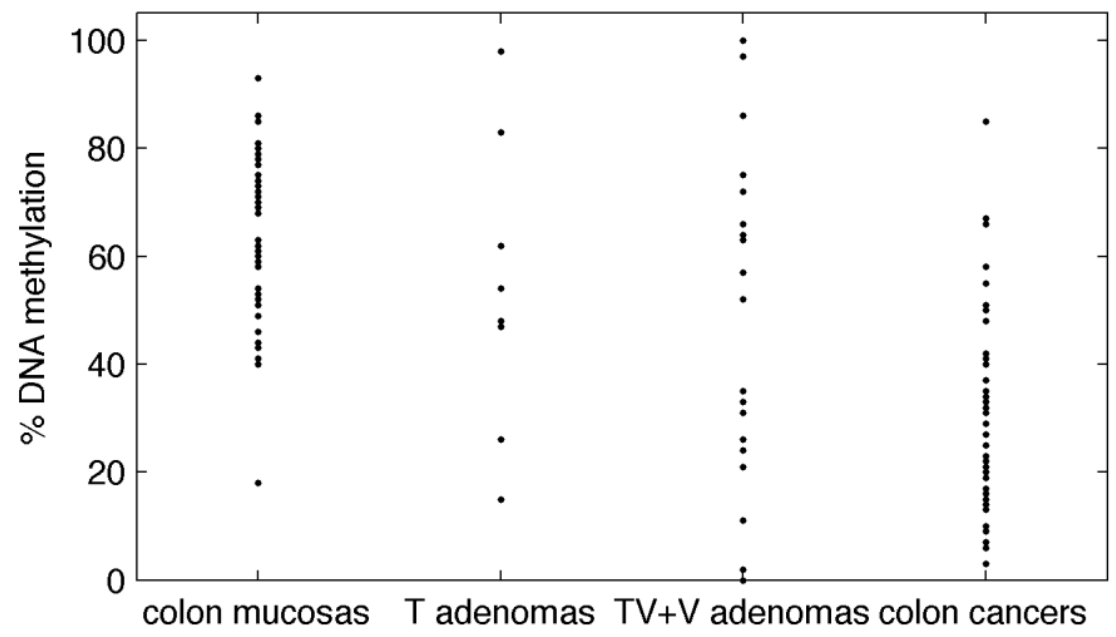

Table 1. Clinical features of the analyzed samples.

\begin{tabular}{cccccccc}
\hline Samples & Sex & Age & Site & $\begin{array}{c}\text { Histological } \\
\text { type }\end{array}$ & $\begin{array}{c}\text { Grade of } \\
\text { dysplasia }\end{array}$ & $\begin{array}{c}\text { \% precancerous } \\
\text { cells }\end{array}$ & $\begin{array}{c}\text { \% DNA } \\
\text { methylation }^{\dagger}\end{array}$ \\
\hline T1 & M & 62 & Right colon & T & LG & 60 & 62 \\
T3 & M & 75 & Sigmoid & TV & LG & 100 & 52 \\
T4* & F & 69 & Right colon & TV & LG & 100 & 0 \\
T5* & F & 69 & Right colon & TV & LG & 100 & 24 \\
T6 & M & 70 & Sigmoid & V & LG & 100 & 63 \\
T7 & M & 70 & Sigmoid & TV & LG & 100 & 66 \\
T10 & M & 56 & Right colon & TV & LG & 60 & 97 \\
T11 & M & 63 & Left colon & TV & LG & 100 & 11 \\
T12 & F & 51 & Rectum & V & LG & 80 & 0 \\
T13 & F & 62 & Left colon & T & LG & 100 & 83 \\
T14 & F & 19 & Coloproctectomy & T & LG & 100 & 54 \\
T15 & F & 19 & Coloproctectomy & TV & LG & 100 & 2 \\
T16 & M & 60 & Sigmoid & T & LG & 100 & 47 \\
T17 & F & 56 & Coloproctectomy & T & LG & 100 & 98 \\
T18 & M & 70 & Right colon & T & LG & 100 & 48 \\
T19 & M & 67 & Coloproctectomy & V & LG and HG & 100 & 75 \\
T20 & M & 59 & Coloproctectomy & T & LG & 100 & 15 \\
M21 & F & 56 & Colon & V & LG & 90 & 57 \\
M22 & F & 58 & Colon & TV & LG & 85 & 52 \\
M24 & M & 60 & Colon & TV & HG & 90 & 31 \\
M25 & M & 78 & Colon & T & LG & 85 & 48 \\
\hline
\end{tabular}


Table 1. Cont.

\begin{tabular}{cccccccc}
\hline M26 & M & 61 & Rectum & V & LG & 90 & 33 \\
M27 & M & 70 & Colon & V & HG & 90 & 24 \\
M40 & F & 21 & Colon & T & LG & 90 & 26 \\
K3 & M & 80 & Rectum & - & LG & n.d. & 28 \\
K4 & F & 56 & Rectum & V & LG & n.d. & 26 \\
K8 & M & 63 & Rectum & - & LG & n.d. & 6 \\
K9a\# & F & 48 & Rectum & - & LG & n.d. & 50 \\
K9b\# & F & 48 & Rectum & - & LG & n.d. & 16 \\
K10 & M & 61 & Rectum & - & HG & n.d. & 49 \\
K11 & M & 63 & n.d. & - & LG & n.d. & 102 \\
K13 & M & 39 & Rectum & - & HG & n.d. & 59 \\
K14 & M & 84 & Rectum & V & HG & n.d. & 86 \\
K15 & M & 53 & Rectum & - & LG & n.d. & 40 \\
K16 & M & 46 & Rectum & - & LG & n.d. & 93 \\
K17 & M & 67 & Rectum & - & LG & n.d. & 30 \\
K18 & M & 72 & Rectum & - & LG & n.d. & 37 \\
K19 & M & 58 & Rectum & - & LG & n.d. & 43 \\
K20 & M & 28 & Rectum & - & HG & n.d. & 82 \\
K21 & F & 56 & Sigmoid & V & LG & n.d. & 64 \\
K22 & F & 37 & Rectum & V & LG & n.d. & 100 \\
K23 & M & 59 & Sigmoid & V & LG & n.d. & 21 \\
K24 & F & 73 & Sigmoid & V & LG & n.d. & 72 \\
K25 & F & 77 & Rectum & V & LG & n.d. & 35 \\
\hline
\end{tabular}

$\mathrm{T}=$ tubulous; $\mathrm{TV}=$ tubulo-villous; $\mathrm{V}=$ villous; $\mathrm{LG}=$ low grade; $\mathrm{HG}=$ high grade; $\dagger$ Mean of two duplicates; n.d. $=$ not detailed by pathologist; $* \#$ Two polyps from a same patient; - excluded.

\subsection{Hypomethylation of BAGE Loci and Histological Features}

The prevalence of DNA hypomethylation in BAGE loci was higher in tubulo-villous and villous adenomas (50\%) than in tubulous adenomas $(22.2 \%)$ (Table 2-a). Such a difference was not significant $(\mathrm{p}=0.15)$; however, the comparison between these two groups of tissues should be done in a larger number of samples. We analyzed 31 adenomas out of 44: 13 samples were excluded because they had been classified by the pathologist in two groups (tubulous and villous) instead of three (tubulous, tubulo-villous and villous) and we thought this might introduce a bias. No correlation was found between the grade of dysplasia and loss of DNA methylation (Wilcoxon test, $\mathrm{p}=0.52$ ). However, most adenomas had a low grade of dysplasia.

Afterwards, we compared the hypomethylation frequency in colon adenomas (data from this work) with the hypomethylation of $B A G E$ loci in colon cancers and healthy colon mucosas (data from our earlier study [6]). Paired colon cancers and healthy mucosas were obtained from a different cohort of patients with respect to adenomas. Raw data are represented in Figure 1. The prevalence of hypomethylation was very low in healthy colon mucosas (5.6\%), increased in colon adenomas (43.2\%), particularly in tubulo-villous and villous adenomas $(50 \%)$, and rose even higher in colon cancers (83.0\%) (Table 2-a; Figure 2). When we compared these tissues two by two, the enrichment in 
hypomethylated samples was significant in cancers versus adenomas in general and also in tubulovillous and villous adenomas versus healthy mucosas (Table 2-b). Confidence intervals were wider in adenomas than in colon cancers or mucosas: this variability points up the heterogeneity of DNA methylation in colon adenomas which represent a transition between normal and frankly malignant tissues.

Figure 2. Frequency of hypomethylated DNA ( $<42 \%$ according to [6]) and their $95 \%$ confidence intervals. Data in healthy mucosas and colon cancers are from [6]. $\mathrm{T}$ are tubulous, TV are tubulo- villous and V are villous adenomas.

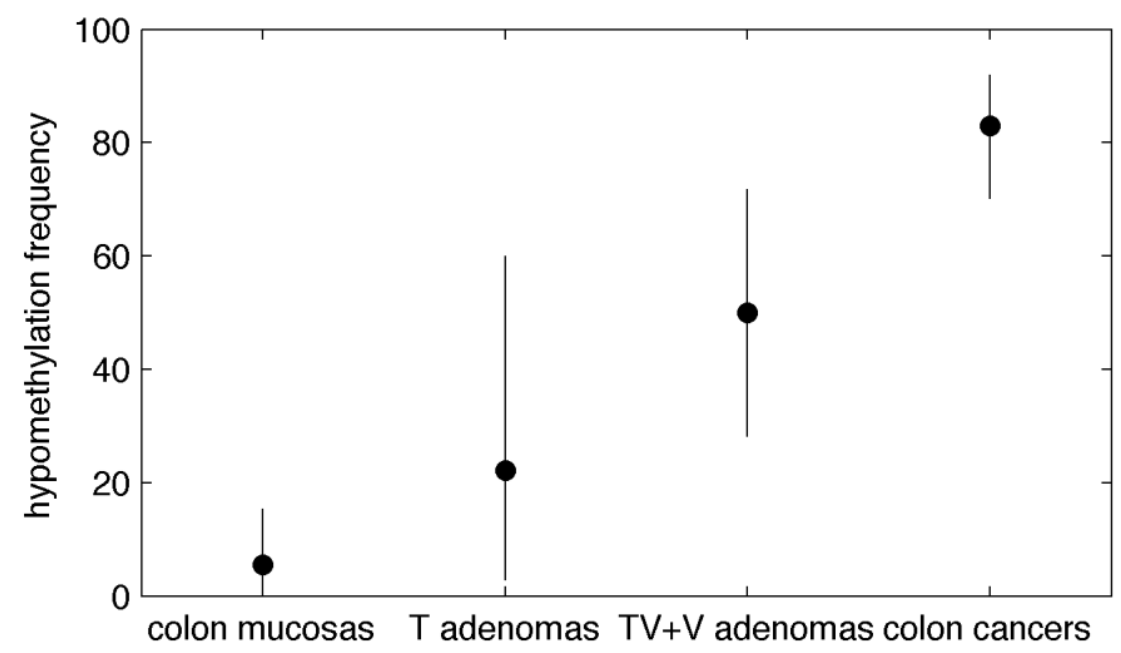

Table 2. (a) Hypomethylation frequency in various tissues.

\begin{tabular}{llll}
\hline Tissues & Frequencies & Min\% (2) & Max\% (2) \\
\hline mucosas (1) & $(3 / 54) 5.6 \%$ & 1.2 & 15.4 \\
cancers (1) & $(44 / 53) 83.0 \%$ & 70.2 & 91.9 \\
adenomas & $(19 / 44) 43.2 \%$ & 28.3 & 59.0 \\
T adenomas & $(2 / 9) 22.2 \%$ & 2.8 & 60.0 \\
TV + V adenomas & $(11 / 22) 50.0 \%$ & 28.2 & 71.8 \\
\hline
\end{tabular}

Table 2. (b) Enrichment test of hypomethylated samples.

\begin{tabular}{ll}
\hline Tissues & p-Values \\
\hline adenomas vs mucosas & $7 \times 10^{-5}$ \\
cancers vs adenomas & $1.4 \times 10^{-6}$ \\
TV+V adenomas vs mucosas & $2.7 \times 10^{-5}$ \\
TV+V adenomas vs cancers & $4.7 \times 10^{-3}$ \\
T adenomas vs mucosas & 0.15 \\
T adenomas vs cancers & $6.1 \times 10^{-4}$ \\
TV+V adenomas vs T adenomas & 0.15 \\
\hline
\end{tabular}

(1) data from [6]; (2) 95\% confidence interval; $\mathrm{T}=$ tubulous; $\mathrm{TV}=$ tubulo-villous; $\mathrm{V}=$ villous. 


\section{Experimental Section}

\subsection{DNA Samples}

Forty-four colorectal adenomas were obtained from 40 patients (Table 1). All subjects provided written informed consent. Frozen tissues were used partly for DNA extraction and partly for histopathological inspection. DNA was quantified spectrophotometrically (Thermo Scientific NanoDrop 1000 Spectrophotometer, Wilmington, DE, USA): samples having a ratio $\mathrm{A}_{260 / 280} \geq 1.8$ were used for the DNA methylation analysis.

\subsection{DNA Methylation Analysis}

DNA methylation in BAGE loci was analyzed according to a previously described combined bisulphite restriction assay (COBRA) protocol [5]. Briefly, genomic DNA was treated with sodium bisulphite and BAGE loci were amplified in two consecutive PCR with a set of nested primers and $5 \mathrm{U}$ of Taq DNA Polymerase (MP Biomedicals, Illkirch, France). Primers in the first PCR were BAGE.BS+32f (TttagaggaTTaggagaagggggagT) and BAGE.BS+1540r (AcctAccaAttAAcattAttActA acattA). Primers in the nested PCR were BAGE.BS99f (gatggtggtggTaaTagagatggT) and BAGE.BS+1371r (ccttaAAcaAtAtaAacctAataA). In the first PCR we used approximately $15 \mathrm{ng}$ of genomic DNA as template, whereas in the nested PCR, we used $2.5 \mu \mathrm{L}$ of the first PCR mixture. The PCR program was $94{ }^{\circ} \mathrm{C}$ for $2 \mathrm{~min}$, followed by five cycles of $\left(94{ }^{\circ} \mathrm{C}\right.$ for $1 \mathrm{~min}, 55{ }^{\circ} \mathrm{C}$ for 2 min and $72{ }^{\circ} \mathrm{C}$ for $\left.3 \mathrm{~min}\right)$. Then 25 cycles of $\left(94{ }^{\circ} \mathrm{C}\right.$ for $0.5 \mathrm{~min}, 55^{\circ} \mathrm{C}$ for $2 \mathrm{~min}$ and $72{ }^{\circ} \mathrm{C}$ for $1.5 \mathrm{~min}$ and a final elongation at $72{ }^{\circ} \mathrm{C}$ ) for $10 \mathrm{~min}$. The products of three nested PCRs were purified using the QIAquick PCR Purification kit (QIAGEN, Hilden, Germany) and quantified spectrophotometrically. The purified PCR products (700 ng) were then digested with $2.5 \mathrm{U}$ of $\mathrm{MboI}$ enzyme (New England Biolabs) 2 hours at $37{ }^{\circ} \mathrm{C}$ and separated by electrophoresis on a $2 \%$ agarose gel in SB buffer [8.2 mM $\mathrm{NaOH}, 5 \mathrm{mM}$ borate]. Images were captured with an Infinity 3000 imaging system (Vilber Lourmat, Marne-la-Vallée, France). This COBRA assay is based upon simultaneous amplification and digestion of several BAGE loci that produces a complex pattern of DNA bands. Two bands of $287 \mathrm{bp}$ and $241 \mathrm{bp}$ are diagnostic: their relative intensity is correlated with $B A G E$ methylation. The relative intensity of the diagnostic bands was measured in individual samples and in each point of a standard curve that allowed us to calculate the methylation percentages. The standard curve was obtained by combining increasing amounts of sperm DNA (approximately 0\% DNA methylation) and M.SssI-treated (New England Biolabs) DNA (100\% DNA methylation). All experiments were done in duplicate. Methylation values over $100 \%$ have two explanations. First, polymorphism and/or chromosome rearrangements in the samples may change the relative amount of diagnostic bands. Second, the method used to analyse $B A G E$ methylation has an accuracy of approximately $10 \%$ and this may also account for methylation values over $100 \%$. For a detailed explanation of the method see [5].

\subsection{Statistical Analysis}

To establish whether DNA methylation was correlated with the age of the patients and with the percentage of precancerous cells (continuous variables), we computed Spearman's correlation 
coefficient and the associated p-value. To test the correlation with sex and dysplasia (categorical variables), Wilcoxon's ranksum test was performed. The correlation between DNA methylation and the location of adenomas (right/left colon, sigmoid, rectum and coloproctectomy) was analyzed with a Kruskal-Wallis test. Since the number of samples in each of the five tissues was small, the confidence intervals for the probabilities of Table 2-a and Figure 2 were established using the binomial distribution, and are hence asymmetrical. Probabilities were compared with Fisher's exact test, either one-sided for the enrichment tests of Table 2-b, or two-sided (see [7] for details). A p-value of less than 0.05 was considered significant.

\section{Conclusions}

Tumor transformation is frequently associated with epigenetic changes: both a global loss of DNA methylation and local increases of DNA methylation in the promoter region of several genes have been reported. Aberrant methylation may contribute to tumor progression by (i) silencing tumor suppressor genes; (ii) activating oncogenes; and (iii) promoting genome instability [8,9]. Detection of aberrant DNA methylation can be used to develop new biological markers that differentiate cancer cells from normal cells [10]. To date, most studies have focused on DNA hypermethylation: several gene promoters are hypermethylated in colorectal cancers and in colorectal adenomas [11-13]. Hypermethylation has been found not only in individual genes, but also in large chromosome regions [14]. Few studies have investigated DNA hypomethylation in colon cancer. Bariol et al., [15] analyzed global DNA methylation levels in a collection of adenomas, hyperplastic polyps, colorectal cancers, and normal mucosa: normal mucosa was more methylated than any cancerous or precancerous lesion. Overall, these findings show that epigenetic changes occur early during tumor transformation.

We showed earlier that $B A G E$ loci were significantly hypomethylated in colon cancers compared to paired healthy mucosas [6]. In the present study, we provide evidence that hypomethylation of $B A G E$ loci was an early event occurring in $43 \%$ of colorectal adenomas. Interestingly, hypomethylation of $B A G E$ loci was frequent (50\%) in tubulo-villous and villous adenomas, these adenomas having a high probability of being transformed into colorectal cancers [16]. In conclusion, hypomethylation of $B A G E$ loci could be used for the diagnosis of colorectal cancers and histologically advanced adenomas. Aberrant hypermethylation of some genes is also more frequent in tubulo-villous and villous adenomas than in tubulous adenomas. Petko et al. [17] observed an increase of hypermethylation in the CDKN2A and MGMT genes, but not in the $M L H 1$ gene in advanced adenomas.

Aberrant methylation of gene promoters may be detected in fecal DNA of patients with colorectal carcinomas and adenomas [17-19]. In colorectal cancers, the rate of colonocyte exfoliation is much higher than in normal mucosa [20]. Exfoliated cells migrate along the gut following the fecal flow and their DNA can be purified from stools. In spite of the presence of normal contaminating squamous cells, significant changes in DNA methylation at gene promoters have been reported [17-19]. The main drawback of using stool DNA to screen the general population is the low sensitivity of single tumor markers. To increase the final sensitivity and specificity, reliable diagnostic assays should combine multiple epigenetic and genetic markers that have been accurately chosen from among the most informative. Likely, a combination of markers based on hyper- and hypomethylation will be more informative because the two events seem to be independent [15]. Among markers based upon 
loss of methylation, $B A G E$ loci are particularly informative. Contrary to LINE sequences that are hypomethylated in $30 \%$ of normal mucosa, $B A G E$ loss of methylation is very specific and also rather sensitive in DNA that has been extracted from biopsies [6]. Thus, the feasibility of detecting $B A G E$ hypomethylation in stools of patients with colorectal cancer and/or precancerous lesions should be evaluated. Another factor to be considered in the development of noninvasive tests to monitor the general population is their cost. To reduce the cost, it will be important to optimize an analytical approach that can be easily implemented in a hospital laboratory and allows the simultaneous analysis of several samples. Pyrosequencing, revealing both point mutations and methylation changes over 100bp-DNA stretches, seems to us an appropriate solution [21]. Kits enabling DNA extraction from stool and bisulfite treatment of genomic DNA can be purchased. Moreover, pyrosequencers host 96 samples per run. This technology, widely used to detect k-ras mutations in cancer, may become a powerful and cost-effective screening method, provided multiple genetic and epigenetic markers are analyzed.

\section{Acknowledgements}

We thank Karine Gordien and Sophie Peries (GSO Tumor Bank Toulouse Hospital) and Blandine Massemin (CRLC, Montpellier, France) for DNA extraction. This work was supported by the "Ligue contre le Cancer" ("Comité de l'Hérault" and "Comité de la Lozère"). E.L. was supported by the "Fondation Jérôme Lejeune" and by the "Fondation pour la Recherche Médicale".

\section{References and Notes}

1. Achkar, E.; Moayyedi, P. Colorectal Cancer Screening with Fecal Occult Blood Testing (FOBT): an international perspective. Am. J. Gastroenterol. 2006, 101, 212.

2. Kinzler, K.W.; Vogelstein, B. Lessons from hereditary colorectal cancer. Cell 1996, 87, 159-170.

3. Ruault, M.; van der Bruggen, P.; Brun, M.E.; Boyle, S.; Roizès, G.; De Sario, A. New BAGE (B melanoma antigen) genes mapping to the juxtacentromeric regions of human chromosomes 13 and 21 have a cancer/testis expression profile. Eur. J. Hum. Genet. 2000, 10, 833-840.

4. Ruault, M.; Ventura, M.; Galtier, N.; Brun, M.E.; Archidiacono, N.; Roizès, G.; De Sario, A. BAGE genes generated by juxtacentromeric reshuffling in the Hominidae lineage are under selective pressure. Genomics 2003, 81, 391-399.

5. Grunau, C.; Sanchez, C.; Ehrlich, M.; van der Bruggen, P.; Hindermann, W.; Rodriguez, C.; Krieger, S.; Dubeau, L.; Fiala, E.; De Sario, A. Frequent DNA hypomethylation of human juxtacentromeric BAGE loci in cancer. Genes Chromosomes Cancer 2005, 43, 11-24.

6. Grunau, C.; Brun, M.E.; Rivals, I.; Selves, J.; Hindermann, W.; Favre-Mercuret, M.; Granier, G.; De Sario, A. BAGE hypomethylation, a new epigenetic biomarker for colon cancer detection. Cancer Epidemiol. Biomarkers Prev. 2008, 17, 1374-1379.

7. Rivals, I.; Personnaz, L.; Taing, L.; Potier, M.C. Enrichment or depletion of a GO category within a class of genes: which test? Bioinformatics 2007, 23, 401-407.

8. Das, P.M.; Singal, R. DNA Methylation and Cancer. J. Clin. Oncol. 2004, 22, 4632-4642. 
9. Hoffmann, M.J.; Schulz, W.A. Causes and consequences of DNA hypomethylation in human cancer. Biochem. Cell Biol. 2005, 83, 296-321.

10. Paluszczak, J.; Baer-Dubowska, W. Epigenetics diagnostics of cancer-the application of DNA methylation markers. J. Appl. Genet. 2006, 47, 365-375.

11. Toyota, M.; Ohe-Toyota, M.; Ahuja, N.; Issa, J.P. Distinct genetic profiles in colorectal tumors with or without the $\mathrm{CpG}$ island methylator phenotype. Proc. Natl. Acad. Sci. USA 2000, 97, $710-715$.

12. Grady, W.M. Epigenetic events in the colorectum and in colon cancer. Biochem. Soc. Trans. 2005, 33, 684-688.

13. Frigola, J.; Song, J.; Stirzaker, C.; Hinshelwood, R.A.; Peinado, M.A.; Clark, S.J. Epigenetic remodelling in colorectal cancer result in coordinate gene suppression across an entire chromosome band. Nat. Genet. 2006, 38, 504-506.

14. Frigola, J.; Song, J.; Stirzaker, C.; Hinshelwood, R.A.; Peinado, M.A.; Clark, S.J. Epigenetic remodeling in colorectal cancer results in coordinate gene suppression across an entire chromosome band. Nat. Genet. 2006, 38, 540-549.

15. Bariol, C.; Suter, C.; Cheong, K.; Ku, S.L.; Meagher, A.; Hawkins, N.; Ward, R. The relationship between hypomethylation and $\mathrm{CpG}$ island methylation in colorectal neoplasia. Am. J. Pathol. 2003, 162, 1043-1045.

16. Haot, J.; Jouret, A. Colonic dysplasia in polyps and flat lesions. Acta Endoscopica 2000, 30, $145-152$.

17. Petko, Z.; Ghiassi, M.; Shuber, A.; Gorham, J.; Smalley, W.; Washington, M.K.; Schultenover, S.; Gautam, S.; Markowitz, S.D.; Grady, W.M. Aberrantly methylated CDKN2A, MGMT, and MLH1 in colon polyps and in fecal DNA from patients with colorectal polyps. Clin. Cancer Res. 2005, 11, 1203-1209.

18. Müller, H.M.; Oberwalder, M.; Fiegl, H.; Morandell, M.; Goebel, G.; Zitt, M.; Mühlthaler, M.; Ofner, D.; Margreiter, R.; Widschwendter, M. Methylation changes in faecal DNA: a marker for colorectal cancer screening? Lancet 2004, 363, 1283-1285.

19. Huang, Z.H.; Li, L.H.; Yang, F.; Wang, J.F. Detection of aberrant methylation in fecal DNA as a molecular screening tool for colorectal cancer and precancerous lesions. World J. Gastroenterol. 2007, 13, 950-954.

20. Loktionov, A. Cell exfoliation in the human colon: myth, reality and implications for colorectal cancer screening. Int. J. Cancer 2007, 120, 2281-2289.

21. Tost, J. DNA methylation: An introduction to the biology and the disease-associated changes of a promising biomarker. Methods Mol. Biol. 2009, 507, 3-20.

(C) 2009 by the authors; licensee Molecular Diversity Preservation International, Basel, Switzerland. This article is an open-access article distributed under the terms and conditions of the Creative Commons Attribution license (http://creativecommons.org/licenses/by/3.0/). 